\title{
Generalized Volterra functions, its integral representations and applications to the Mathieu-type series
}

\author{
Khaled Mehrez ${ }^{\mathrm{a}, \mathrm{b}, *}$, Sergei M. Sitnik ${ }^{\mathrm{c}}$ \\ a Département de Mathématiques, Issat Kasserine, Université de Kairouan, Tunisia \\ b Département de Mathématiques, Facultée des sciences de Tunis, Université Tunis El Manar, Tunisia \\ ${ }^{\mathrm{c}}$ Belgorod State National Research University (BSU), Belgorod, Russia
}

\section{A R T I C L E I N F O}

\section{JEL classification:}

$11 \mathrm{M} 35$

33D05

33B15

26A51

Keywords:

Generalized Volterra functions Complete monotonicity

Log-convex functions

Turán type inequalities

Mathieu-type series

\begin{abstract}
A B S T R A C T
In this paper we introduce the new class of generalized Volterra functions. We prove some integral representations for them via Fox-Wright $\mathrm{H}$-functions and Meijer G-functions. From positivity conditions on the weight in these representations, we found sufficient conditions on parameters of the generalized Volterra function to prove its complete monotonicity. As applications, a Turán type inequality for generalized Volterra functions is derived, infinite integral of some special functions are expressed in terms of the generalized Volterra functions and closed-form integral representations for a family of convergent Mathieu-type series defined in terms of generalized Volterra functions are established.
\end{abstract}

(c) 2018 Elsevier Inc. All rights reserved.

\section{Introduction}

Consider definitions of classical Volterra and related functions as stated in [1, p. 217]:

$$
\begin{aligned}
& v(x)=\int_{0}^{\infty} \frac{x^{t}}{\Gamma(t+1)} d t, \\
& v(x, \alpha)=\int_{0}^{\infty} \frac{x^{t+\alpha}}{\Gamma(t+\alpha+1)} d t, \\
& \mu(x, \beta)=\int_{0}^{\infty} \frac{x^{t} t^{\beta}}{\Gamma(t+1) \Gamma(\beta+1)} d t \\
& \mu(x, \beta, \alpha)=\int_{0}^{\infty} \frac{x^{t+\alpha} t^{\beta}}{\Gamma(t+\alpha+1) \Gamma(\beta+1)} d t,
\end{aligned}
$$

where $\alpha, \beta>-1$ and $x>0$, but some particular notations are usually adopted in special cases

$$
\alpha=\beta=0, \quad v(x)=\mu(x, 0,0)
$$

\footnotetext{
* Corresponding author at: Département de Mathématiques, Issat Kasserine, Université de Kairouan, Tunisia.

E-mail addresses: k.mehrez@yahoo.fr (K. Mehrez), Sitnik@bsu.edu.ru (S.M. Sitnik).
} 


$$
\begin{aligned}
& \alpha \neq 0, \beta=0, \quad v(x, \alpha)=\mu(x, 0, \alpha) \\
& \alpha=0, \beta \neq 0, \quad \mu(x, \beta)=\mu(x, \beta, 0) .
\end{aligned}
$$

Volterra functions were introduced by Vito Volterra in 1916. Its theory was thoroughly developed by Mhitar M. Dzhrbashyan, his own and his coathors results were summed up in the monograph [2] in 1966. In this book many important results on Volterra functions, known and new, were gathered and introduced. Many results on Volterra functions were also gathered in two books of Apelblat [3,4], for important application cf. also [7].

In this paper we define the new class of generalized Volterra functions $V_{p, q}^{\alpha, \beta}\left[\right.$.] with $p$ numerator parameters $\alpha_{1}, \ldots, \alpha_{p}$ and $q$ denominator parameters $\beta_{1}, \ldots, \beta_{q}$, by

$$
V_{p, q}^{\alpha, \beta}\left[\begin{array}{c}
\left(b_{1}, B_{1}\right), \ldots,\left(b_{q}, B_{q}\right) \\
\left(a_{1}, A_{1}\right), \ldots,\left(a_{p}, A_{p}\right)
\end{array} \mid x\right]=V_{p, q}^{\alpha, \beta}\left[\begin{array}{c}
\left(\beta_{q}, B_{q}\right) \\
\left(a_{p}, A_{p}\right)
\end{array} \mid x\right]=\int_{0}^{\infty} \frac{\prod_{i=1}^{p} \Gamma\left(A_{i} t+a_{i}\right)}{\prod_{j=1}^{q} \Gamma\left(B_{j} t+b_{j}\right)} \frac{x^{t+\alpha} t^{\beta}}{\Gamma(\beta+1)} d t
$$

where

$$
\left(\alpha, \beta>-1, x>0, A_{i}, B_{j}>0, a_{i}, b_{j} \geq 0,(i=1, \ldots, p ; j=1, \ldots, q)\right) .
$$

For the special case $x=1 / e, \beta=s-1$ the function (1.6) up to the constant reduces to the function

$$
\tilde{V}_{p, q}^{\alpha, \beta}\left[\begin{array}{c}
\left(\beta_{q}, B_{q}\right) \\
\left(a_{p}, A_{p}\right)
\end{array} \mid p, s\right]=\int_{0}^{\infty} \frac{\prod_{i=1}^{p} \Gamma\left(A_{i} t+a_{i}\right)}{\prod_{j=1}^{q} \Gamma\left(B_{j} t+b_{j}\right)} e^{-p t} t^{s-1} d t,
$$

which is interesting and important as a simultaneous expression for Laplace and Mellin transforms for the gamma-function ratio.

The paper is organized as follows. In Section 2 we prove several integral representations for the new class of the generalized Volterra function via Fox-Wright functions and the Laplace transform. Various new facts regarding the generalized Volterra function are proved, including complete monotonicity property, log-convexity in upper parameters, and a Turán type inequality. In Section 3 closed-form integral expressions are derived for a family of convergent Mathieu-type series and its alternating variant when terms contain the generalized Volterra function.

\section{Integral representations for the generalized Volterra functions}

To formulate our first main result we need a particular case of Fox's H-function defined by

$$
H_{q, p}^{p, 0}\left(\left.z\right|_{\left(A_{1}, a_{1}\right), \ldots,\left(A_{p}, a_{p}\right)} ^{\left(B_{1}, b_{1}\right), \ldots,\left(B_{q}, b_{q}\right)}\right)=H_{q, p}^{p, 0}\left(\left.z\right|_{\left(A_{p}, a_{p}\right)} ^{\left(B_{q}, b_{q}\right)}\right)=\frac{1}{2 i \pi} \int_{\mathcal{L}} \frac{\prod_{i=1}^{p} \Gamma\left(A_{i} s+a_{i}\right)}{\prod_{j=1}^{q} \Gamma\left(B_{k} s+b_{j}\right)} z^{-s} d s,
$$

where $A_{i}, B_{j}>0$ and $a_{i}, b_{j}$ are real. The contour $\mathcal{L}$ can be either the left loop $\mathcal{L}_{-}$starting at $-\infty+i \alpha$ and ending at $-\infty+i \beta$ for some $\alpha<0<\beta$ such that all poles of the integrand lie inside the loop, or the right loop $\mathcal{L}_{+}$starting $\infty+i \alpha$ at and ending $\infty+i \beta$ and leaving all poles on the left, or the vertical line $\mathcal{L}_{i c}, \Re(z)=c$, traversed upward and leaving all poles of the integrand on the left. Denote the rightmost pole of the integrand by $\gamma$ :

Let

$$
\gamma=-\min _{1 \leq i \leq p}\left(a_{i} / A_{i}\right)
$$

$$
\rho=\left(\prod_{i=1}^{p} A_{i}^{A_{i}}\right)\left(\prod_{j=1}^{q} B_{j}^{-B_{j}}\right), \mu=\sum_{j=1}^{q} b_{j}-\sum_{i=1}^{p} \alpha_{i}+\frac{p-q}{2}
$$

Existence conditions of Fox's H-function under each choice of the contour $\mathcal{L}$ have been thoroughly considered in the book [16]. Let $z>0$ and under conditions:

$$
\sum_{i=1}^{p} A_{j}=\sum_{j=1}^{q} B_{j}, \quad \rho \leq 1
$$

we get that the function $H_{q, p}^{p, 0}(z)$ exists by means of [16, Theorem 1.1], if we choose $\mathcal{L}=\mathcal{L}_{+}$or $\mathcal{L}=\mathcal{L}_{i c}$ under the additional restriction $\mu>1$. Only the second choice of the contour ensures the existence of the Mellin transform of $H_{q, p}^{p, 0}(z)$, see [16, Theorem 2.2]. In [11, Theorem 6], the authors extend the condition $\mu>1$ to $\mu>0$ and proved that the function $H_{q, p}^{p, 0}(z)$ is of compact support.

Theorem 1. Let $\alpha, \beta>-1$. Assume that $\mu>0$, and $\sum_{j=1}^{p} A_{j}=\sum_{k=1}^{q} B_{k}$. Then the following integral representation for the generalized Volterra function (1.6)

$$
V_{p, q}^{\alpha, \beta}\left[\begin{array}{c}
\left(a_{p}, A_{p}\right) \\
\left(\beta_{q}, B_{q}\right)
\end{array} \mid x\right]=\int_{0}^{\rho} H_{q, p}^{p, 0}\left(\left.t\right|_{\left(A_{p}, \alpha_{p}\right)} ^{\left(B_{q}, \beta_{q}\right)}\right) \frac{x^{\alpha} d t}{t \log ^{\beta+1}(1 /(t x))},
$$


holds true for all $x \in(0,1)$.

Proof. By using the Mellin transform for the Fox's H-function $H_{q, p}^{p, 0}(z)$ [11, Theorem 6]:

$$
\frac{\prod_{i=1}^{p} \Gamma\left(A_{i} t+\alpha_{i}\right)}{\prod_{k=1}^{q} \Gamma\left(B_{k} t+\beta_{k}\right)}=\int_{0}^{\rho} H_{q, p}^{p, 0}\left(\left.z\right|_{\left(A_{p}, \alpha_{p}\right)} ^{\left(B_{q}, \beta_{q}\right)}\right) z^{t-1} d t, \Re(t)>\gamma,
$$

we obtain

$$
\begin{aligned}
V_{p, q}^{\alpha, \beta}\left[\begin{array}{c}
\left(a_{p}, A_{p}\right) \\
\left(\beta_{q}, B_{q}\right)
\end{array} \mid x\right] & =\int_{0}^{\infty} \frac{\prod_{i=1}^{p} \Gamma\left(A_{i} t+a_{i}\right)}{\prod_{j=1}^{q} \Gamma\left(B_{j} t+b_{j}\right)} \frac{x^{t+\alpha} t^{\beta}}{\Gamma(\beta+1)} d t \\
& =\int_{0}^{\infty} \int_{0}^{\rho} H_{q, p}^{p, 0}\left(\left.z\right|_{\left(A_{p}, \alpha_{p}\right)} ^{\left(B_{q}, \beta_{q}\right)}\right) \frac{x^{t+\alpha} z^{t-1} t^{\beta}}{\Gamma(\beta+1)} d t d z \\
& =\int_{0}^{\rho} H_{q, p}^{p, 0}\left(\left.z\right|_{\left(A_{p}, \alpha_{p}\right)} ^{\left(B_{q}, \beta_{q}\right)}\right)\left(\int_{0}^{\infty} x^{t} z^{t} t^{\beta} d t\right) \frac{x^{\alpha} z^{-1}}{\Gamma(\beta+1)} d z \\
& =\int_{0}^{\rho} H_{q, p}^{p, 0}\left(\left.z\right|_{\left(A_{p}, \alpha_{p}\right)} ^{\left(B_{q}, \beta_{q}\right)}\right)\left(\int_{0}^{\infty} t^{\beta} e^{-t} d t\right) \frac{x^{\alpha} z^{-1}}{\Gamma(\beta+1) \log ^{\beta+1}(1 /(x z))} d z \\
& =\int_{0}^{\rho} H_{q, p}^{p, 0}\left(\left.z\right|_{\left(A_{p}, \alpha_{p}\right)} ^{\left(B_{q}, \beta_{q}\right)}\right) \frac{x^{\alpha} z^{-1}}{\log ^{\beta+1}(1 /(x z))} d z .
\end{aligned}
$$

This completes the proof of Theorem 1.

Remark 1. The special case for which the H-function reduces to the Meijer G-function is when $A_{1}=\cdots=A_{p}=B_{1}=\cdots=$ $B_{q}=A, A>0$. In this case,

$$
H_{q, p}^{m, n}\left(\left.z\right|_{\left(A, a_{p}\right)} ^{\left(A, b_{q}\right)}\right)=\frac{1}{A} G_{p, q}^{m, n}\left(\left.z^{1 / A}\right|_{\mathbf{a}_{\mathbf{p}}} ^{\mathbf{b}_{\mathbf{q}}}\right)
$$

where $\mathbf{a}_{\mathbf{p}}=\left(a_{1}, \ldots, a_{p}\right)$ and $\mathbf{b}_{\mathbf{q}}=\left(b_{1}, \ldots, b_{q}\right)$. So we get

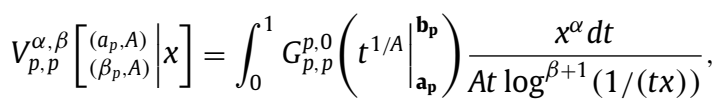

for all $x \in(0,1)$ and $\alpha, \beta>-1$.

Let us note that the special case of the Meijer G-function $G_{p, p}^{p, 0}(\cdot)$ from (2.14) is very important for applications. Due to it in [10] it was proposed to name the function $G_{p, p}^{p, 0}(\cdot)$ as Meijer-Nørlund one, due to important results of Nørlund for this function.

We denote the ratio of gamma-functions by

$$
\psi_{n, m}=\frac{\prod_{i=1}^{p} \Gamma\left(\alpha_{i}+(n+m) A_{i}\right)}{\prod_{j=1}^{q} \Gamma\left(\beta_{j}+(n+m) B_{j}\right)}, n, m \in \mathbb{N}_{0}
$$

In [22, Corollary 1] it was proved that the function $H_{q, p}^{p, 0}(z)$ is non-negative on $(0, \rho)$ if

$$
\left(H_{1}^{n}\right): \psi_{n, 2}<\psi_{n, 1} \text { and } \psi_{n, 1}^{2}<\psi_{n, 0} \psi_{n, 2} \text {, for all } n \in \mathbb{N}_{0} \text {. }
$$

In addition, it was proved that the H-function $H_{p, p}^{p, 0}\left[\left.t\right|_{\left(A, \alpha_{p}\right)} ^{\left(A, \beta_{p}\right)}\right]$ is non-negative, if

$$
\left(H_{2}\right): 0<\alpha_{1} \leq \cdots \leq \alpha_{p}, 0<\beta_{1} \leq \cdots \leq \beta_{p}, \sum_{j=1}^{k} \beta_{j}-\sum_{j=1}^{k} \alpha_{j} \geq 0 \text {, for } k=1, \ldots, p .
$$

Corollary 1. Suppose that conditions $\left(H_{2}\right)$ are satisfied. Then the function

$$
A \mapsto V_{p, p}^{\alpha, \beta}\left[\begin{array}{c}
\left(a_{p}, A\right) \\
\left(\beta_{p}, A\right)
\end{array} \mid x\right],
$$

is log-convex on $[0, \infty)$. Furthermore, the following Turán type inequality

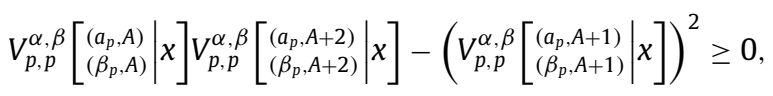

holds true.

Proof. Rewriting the integral representation (2.14) in the following form

$$
V_{p, p}^{\alpha, \beta}\left[\begin{array}{c}
\left(a_{p}, A\right) \\
\left(\beta_{p}, A\right)
\end{array} \mid x\right]=\int_{0}^{1} G_{p, p}^{p, 0}\left(\left.t\right|_{\mathbf{a}_{\mathbf{p}}} ^{\mathbf{b}_{\mathbf{p}}}\right) \frac{\chi^{\alpha} d t}{t(\log (1 / x)+A \log (1 / t))^{\beta+1}},
$$


let us recall the Rogers-Hölder-Riesz inequality [23, p. 54], that is

$$
\int_{a}^{b}|f(t) g(t)| d t \leq\left[\int_{a}^{b}|f(t)|^{p} d t\right]^{1 / p}\left[\int_{a}^{b}|g(t)|^{p} d t\right]^{1 / q}
$$

where $p \geq 1, \frac{1}{p}+\frac{1}{q}=1, f$ and $g$ are real functions defined on $(a, b)$ and $|f|^{p},|g|^{q}$ are integrable functions on $(a, b)$. From the Rogers-Hölder-Riesz inequality again and integral representation (2.16) using the fact that the function $A \mapsto \frac{1}{(a+b A)^{\beta+1}}, a \geq$ $0, b>0$ is log-convex on $[0, \infty)$ we derive that for $A_{1}, A_{2}>0$ and $\lambda \in[0,1]$,

$$
\begin{aligned}
& V_{p, p}^{\alpha, \beta}\left[\begin{array}{c}
\left(a_{p}, \lambda A_{1}+(1-\lambda) A_{2}\right) \\
\left(\beta_{p}, \lambda A_{1}+(1-\lambda) A_{2}\right)
\end{array} \mid x\right]=\int_{0}^{1} G_{p, p}^{p, 0}\left(\left.t\right|_{\mathbf{a}_{\mathbf{p}}} ^{\mathbf{b}_{\mathbf{p}}}\right) \frac{x^{\alpha} d t}{t\left(\log (1 / x)+\log (1 / t)\left(A_{1}+(1-\lambda) A_{2}\right)\right)^{\beta+1}} \\
& \leq \int_{0}^{1} G_{p, p}^{p, 0}\left(\left.t\right|_{\mathbf{a}_{\mathbf{p}}} ^{\mathbf{b}_{\mathbf{p}}}\right) \frac{x^{\alpha} d t}{t\left(\log (1 / x)+A_{1} \log (1 / t)\right)^{\lambda(\beta+1)}\left(\log (1 / x)+A_{2} \log (1 / t)\right)^{(1-\lambda)(\beta+1)}} \\
& \leq \int_{0}^{1}\left[G_{p, p}^{p, 0}\left(\left.t\right|_{\mathbf{a}_{\mathbf{p}}} ^{\mathbf{b}_{\mathbf{p}}}\right) \frac{x^{\alpha}}{t\left(\log (1 / x)+A_{1} \log (1 / t)\right)^{\beta+1}}\right]^{\lambda} \\
& \cdot\left[G_{p, p}^{p, 0}\left(\left.t\right|_{\mathbf{a}_{\mathbf{p}}} ^{\mathbf{b}_{\mathbf{p}}}\right) \frac{x^{\alpha}}{t\left(\log (1 / x)+A_{1} \log (1 / t)\right)^{\beta+1}}\right]^{1-\lambda} d t \\
& \leq\left[\int_{0}^{1} G_{p, p}^{p, 0}\left(\left.t\right|_{\mathbf{a}_{\mathbf{p}}} ^{\mathbf{b}_{\mathbf{p}}}\right) \frac{x^{\alpha} d t}{t\left(\log (1 / x)+A_{1} \log (1 / t)\right)^{\beta+1}}\right]^{\lambda} \\
& \cdot\left[\int_{0}^{1} G_{p, p}^{p, 0}\left(\left.t\right|_{\mathbf{a}_{\mathbf{p}}} ^{\mathbf{b}_{\mathbf{p}}}\right) \frac{x^{\alpha} d t}{t\left(\log (1 / x)+A_{2} \log (1 / t)\right)^{\beta+1}}\right]^{1-\lambda} \\
& =\left(V_{p, p}^{\alpha, \beta}\left[\begin{array}{c}
\left(a_{p}, A_{1}\right) \\
\left(\beta_{p}, A_{1}\right)
\end{array} \mid x\right]\right)^{\lambda}\left(V_{p, p}^{\alpha, \beta}\left[\begin{array}{c}
\left(a_{p}, A_{2}\right) \\
\left(\beta_{p}, A_{2}\right)
\end{array} \mid x\right]\right)^{1-\lambda} .
\end{aligned}
$$

This implies that the function

$$
A \mapsto V_{p, p}^{\alpha, \beta}\left[\begin{array}{c}
\left(a_{p}, A\right) \\
\left(\beta_{p}, A\right)
\end{array} \mid x\right]
$$

is $\log$-convex on $(0, \infty)$. Now let us go to the Turán type inequality (2.15). Choosing $A_{1}=A, A_{2}=A+2$ and $\lambda=\frac{1}{2}$ in the above inequality we get the desired result.

Corollary 2. Suppose that assumptions stated in Theorem 1 and conditions $\left(H_{1}^{n}\right)$ are satisfied. Then the following inequality for the generalized Volterra function

$$
V_{p, q}^{\alpha, \beta_{1}}\left[\begin{array}{c}
\left(a_{p}, A_{p}\right) \\
\left(b_{q}, B_{q}\right)
\end{array} \mid x\right] V_{p, q}^{\alpha, \beta_{2}}\left[\begin{array}{c}
\left(a_{p}, A_{p}\right) \\
\left(b_{q}, B_{q}\right)
\end{array} \mid x\right] \leq \frac{\prod_{i=1}^{p} \Gamma\left(a_{i}\right)}{\prod_{j=1}^{q} \Gamma\left(b_{i}\right)} V_{p, q}^{\alpha, \beta_{1}+\beta_{2}}\left[\begin{array}{c}
\left(a_{p}, A_{p}\right) \\
\left(b_{q}, B_{q}\right)
\end{array} \mid x\right]
$$

holds true for all $0<x<1, \alpha, \beta_{1}, \beta_{2}>-1$.

Proof. Recall the Chebyshev integral inequality [23, p. 40]: if $f, g:[a, b] \longrightarrow \mathbb{R}$ are synchronous (both increasing or decreasing) integrable functions, and $p:[a, b] \longrightarrow \mathbb{R}$ is a positive integrable function, then

$$
\int_{a}^{b} p(t) f(t) d t \int_{a}^{b} p(t) g(t) d t \leq \int_{a}^{b} p(t) d t \int_{a}^{b} p(t) f(t) g(t) d t
$$

Note that if $f$ and $g$ are asynchronous (one is decreasing and the other is increasing), then (2.19) is reversed. Let $\beta_{1}, \beta_{2}>-1$ and consider functions $p, f, g:[0, \rho] \longrightarrow \mathbb{R}$ defined by:

$$
p(t)=t^{-1} H_{q, p}^{p, 0}\left(\left.t\right|_{\left(A_{p}, \alpha_{p}\right)} ^{\left(B_{q}, \beta_{q}\right)}\right), f(t)=\frac{1}{(\log (1 / x)+\log (1 / t))^{\beta_{1}}}, \quad g(t)=\frac{1}{(\log (1 / x)+\log (1 / t))^{\beta_{2}}} .
$$

Since the function $p$ is non-negative on $(0, \rho)$ and functions $f$ and $g$ are increasing on $(0, \rho)$, we conclude that the inequality (2.18) holds true by means of the Chebyshev integral inequality (2.19) applied to the Mellin transform of the Fox's H-function 2.11.

Remark 2. Under the conditions $\left(\mathrm{H}_{2}\right)$, the inequality (2.18) reduces to the following inequality

$$
V_{p, p}^{\alpha, \beta_{1}}\left[\begin{array}{c}
\left(a_{p}, A\right) \\
\left(b_{q}, A\right)
\end{array} \mid x\right] V_{p, p}^{\alpha, \beta_{2}}\left[\begin{array}{c}
\left(a_{p}, A\right) \\
\left(b_{p}, A\right)
\end{array} \mid x\right] \leq \frac{\prod_{i=1}^{p} \Gamma\left(a_{i}\right)}{\prod_{j=1}^{q} \Gamma\left(b_{i}\right)} V_{p, q}^{\alpha, \beta_{1}+\beta_{2}}\left[\begin{array}{c}
\left(a_{p}, A\right) \\
\left(b_{p}, A\right)
\end{array} \mid x\right]
$$

holds true for all $0<x<1, \alpha, \beta_{1}, \beta_{2}>-1$. 
Now let us note that Turán type inequalities and connected results on log-convexity/log-concavity for different classes of special functions are very important and have many applications, cf. [8,9,11,13,14,17-21].

Here, and in what follows, we denote the Laplace transform pair for a suitable function $f$ as follows:

$$
F(t)=L f(t) \text {, and } f(t)=L^{-1} F(t),
$$

that is,

$$
L f(t)=\int_{0}^{\infty} e^{-x t} f(x) d x, \text { and } L^{-1} F(t)=\frac{1}{2 i \pi} \int_{\mathrm{Br}} e^{s t} F(s) d s,
$$

where $\mathrm{Br}$ denotes the Bromwich path. Recall that a function $f:(0, \infty) \longrightarrow(0, \infty)$ is called completely monotonic, if $f$ is continuous on $[0, \infty)$, infinitely differentiable on $(0, \infty)$ and satisfies the following inequality:

$$
(-1)^{n} f^{(n)}(x) \geq 0,\left(x>0, n \in \mathbb{N}_{0}=\{0,1,2, \ldots\}\right) .
$$

The celebrated Bernstein Characterization Theorem gives a sufficient condition for the complete monotonicity of a function $f$ in terms of the existence of some non-negative locally integrable function $K(x)(x>0)$, referred to as the spectral function, for which

$$
f(s)=L(K)(s)=\int_{0}^{\infty} e^{-s t} K(t) d t .
$$

Corollary 3. Suppose that conditions of Theorem 1 are satisfied. In addition, assume that conditions $\left(H_{1}^{n}\right)$ are also valid. Then the function $\mathcal{V}_{p, q}^{\alpha, \beta}[x]$ defined by

$$
\mathcal{V}_{p, q}^{\alpha, \beta}\left[\begin{array}{c}
\left(a_{p}, A_{p}\right) \\
\left(b_{q}, B_{q}\right)
\end{array} \mid x\right]=: V_{p, q}^{\alpha, \beta}\left[\begin{array}{c}
\left(a_{p}, A_{p}\right) \\
\left(\beta_{q}, B_{q}\right)
\end{array} \mid e^{-\chi}\right],
$$

is completely monotonic on $(0, \infty)$ for all $\alpha \geq 0$ and $\beta>-1$. Moreover, the function

$$
\mathcal{V}_{p, q}^{\alpha, \beta}\left[\begin{array}{c}
\left(a_{p}, A_{p}\right) \\
\left(b_{q}, B_{q}\right)
\end{array} \mid x\right]
$$

is also completely monotonic on $(0, \infty)$ for each $\alpha \geq 0$ and $\beta>-1$ under the hypothesis $\left(H_{2}\right)$.

Proof. By using the integral representation (2.10), we can write the function $\mathcal{V}_{p, q}^{\alpha, \beta}[x]$ in the following form:

$$
\mathcal{V}_{p, q}^{\alpha, \beta}\left[\begin{array}{c}
\left(a_{p}, A_{p}\right) \\
\left(b_{p}, B_{q}\right)
\end{array} \mid x\right]=e^{-\alpha x} \int_{0}^{\rho} H_{q, p}^{p, 0}\left(\left.t\right|_{\left(A_{p}, \alpha_{p}\right)} ^{\left(B_{q}, \beta_{q}\right)}\right) \frac{d t}{t(x+\log (1 / t))^{\beta+1}}
$$

So, the above representation reveals that $\mathcal{V}_{p, q}^{\alpha, \beta}[x]$ can be written as a product of two completely monotonic functions. This implies that $\mathcal{V}_{p, q}^{\alpha, \beta}[x]$ is completely monotonic on $(0, \infty)$ for each $\alpha \geq 0$ and $\beta>-1$.

As another applications of Theorem 1, infinite integral of some special functions are expressed in terms of the generalized Volterra functions, see the following examples.

Example 1. Suppose that $a_{1}, a_{2}>1$ and $b_{1}, b_{2}>0$. Then the following identity holds:

$$
\int_{0}^{1} \frac{x^{\alpha} t^{a_{2}-2}(1-t)^{b_{1}+b_{2}-1}}{\Gamma\left(b_{1}+b_{2}\right) \log ^{\beta+1}(1 / x t)} 2 F_{1}\left[\begin{array}{c}
a_{2}+b_{2}-a_{1}, b_{1} \\
b_{1}+b_{2}
\end{array} \mid 1-t\right] d t=V_{2,2}^{\alpha, \beta}\left[\begin{array}{l}
\left(a_{1}-1,1\right),\left(a_{2}-1,1\right) \\
\left(b_{1}+a_{1}-1,1\right),\left(b_{2}+a_{2}-1,1\right)
\end{array} \mid x\right], 0<x<1 .
$$

Indeed, by combining the formula (2.10) with the following formula [16, Eq. (1.142)]

$$
H_{2,2}^{2,0}\left(\left.t\right|_{\left(a_{1}-1,1\right),\left(a_{2}-1,1\right)} ^{\left(a_{1}+b_{1}-1,1\right),\left(a_{2}+b_{2}-1,1\right)}\right)=\frac{t^{a_{2}-1}(1-t)^{b_{1}+b_{2}-1}}{\Gamma\left(b_{1}+b_{2}\right)}{ }_{2} F_{1}\left[\begin{array}{l}
a_{2}+b_{2}-a_{2}, b_{1} \\
b_{1}+b_{2}
\end{array} \mid 1-t\right]
$$

we get (2.23).

Example 2. In view of (2.10) and the identity [15, p. 127]

$$
z^{\alpha}(1-z)^{\beta}=\Gamma(\beta+1) H_{1,1}^{1,0}\left(\begin{array}{l}
(1, \alpha+\beta+1) \\
(1, \alpha)
\end{array}\right)=\Gamma(\beta+1) G_{1,1}^{1,0}\left(\left.z\right|_{\alpha} ^{\alpha+\beta+1}\right), 0<z<1,
$$

we deduce that the following equation

$$
\int_{0}^{1} \frac{t^{a-1}(1-t)^{b} x^{\alpha}}{\Gamma(b+1) \log ^{\beta+1}(1 / x t)} d t=V_{1,1}^{\alpha, \beta}[\underset{(a+b+1,1)}{(a, 1)} \mid x], 0<x<1 .
$$

holds true.

In Corollary 4-6, motivated by Eqs. (2.23) and (2.25), we found new integral formulas. In particular, new formula for the Euler-Mascheroni constant $\gamma$. 
Corollary 4. Let $b>0$. Then, the following formula

$$
\int_{0}^{1} \frac{(1-t)^{2 b-1}{ }_{2} F_{1}\left[\begin{array}{l}
b, b \\
2 b
\end{array} \mid 1-t\right]}{\Gamma(2 b) \log (1 / x t)} d t=\frac{1}{b}-\frac{\log (x)}{x^{b}} \operatorname{Ei}(b \log (x)),
$$

is valid for $0<x<1$, where $\operatorname{Ei}(x)$ is the exponential integral function. In particular, we obtain

$$
\begin{aligned}
& \int_{0}^{1} \frac{\log (1 / t) d t}{\log (1 / x)+\log (1 / t)} d t=1-\frac{\log (x)}{x} \operatorname{Ei}(\log (x)), \\
& \int_{0}^{1} \frac{\log (1 / t) d t}{1+\log (1 / t)} d t=1+e E i(-1) .
\end{aligned}
$$

Proof. Putting $a_{1}=a_{2}=2, \beta=0$ and $b_{1}=b_{2}=b>0$ in (2.23), we find

$$
\int_{0}^{1} \frac{(1-t)^{2 b-1}{ }_{2} F_{1}\left[\begin{array}{l}
b, b \\
2 b
\end{array} \mid 1-t\right]}{\Gamma(2 b) \log (1 / x t)} d t=\int_{0}^{\infty} \frac{x^{t}}{(t+b)^{2}} d t
$$

Take into account the following formula [6, Eq. (09), p. 134]

$$
\int_{0}^{\infty} \frac{e^{-p t}}{(t+a)^{n}} d t=\sum_{m=1}^{n-1} \frac{(m-1) !}{(n-1) !} \frac{(-p)^{n-m-1}}{a^{m}}-\frac{(-p)^{n-1}}{(n-1) !} e^{a p} \operatorname{Ei}(-a p), p \geq 0, n \geq 2,|\arg (a)|<\pi,
$$

with (2.29), we deduce the formula (2.26). Now, letting $b=1$ in (2.26) and using the fact

$$
\log (t)=(t-1)_{2} F_{1}\left[\begin{array}{c|c}
1,1 \\
2
\end{array} \mid 1-t\right]
$$

we get (2.27). Finally, setting $x=\frac{1}{e}$ in (2.27). So, the proof of is completes.

Corollary 5. The following formula

$$
\int_{0}^{1} \frac{d t}{t \log ^{\beta+1}(1 / x t)}=\frac{1}{\beta \log ^{\beta}(1 / x)}, \quad 0<x<1,
$$

holds true for all $\beta>0$. In particular, we have

$$
\begin{aligned}
& \int_{0}^{1} \frac{d t}{t(1-\log (t))^{\beta+1}}=\frac{1}{\beta} \\
& \int_{0}^{1} \frac{d t}{t(1-\log (t))^{2}}=1 \\
& \int_{0}^{1} \frac{d t}{t(1-\log (t))^{3 / 2}}=2 .
\end{aligned}
$$

Proof. By (2.25), we get

$$
\int_{0}^{1} \frac{t^{a-1}(1-t)^{b} d t}{\Gamma(b+1) \log ^{\beta+1}(1 / x t)}=\int_{0}^{\infty} \frac{\Gamma(t+a) x^{t} t^{\beta} d t}{\Gamma(\beta+1) \Gamma(t+a+b+1)} .
$$

Letting $a=b=0$ in the above inequality and from (2.44), we obtain

$$
\begin{aligned}
\int_{0}^{1} \frac{d t}{t \log ^{\beta+1}(1 / x t)} & =\int_{0}^{\infty} \frac{t^{\beta-1} x^{t}}{\Gamma(\beta+1)} d t \\
& =\int_{0}^{\infty} \frac{t^{\beta-1} e^{-\log (1 / x) t}}{\Gamma(\beta+1)} d t \\
& =\frac{1}{\beta \log ^{\beta}(1 / x)} .
\end{aligned}
$$

Finally, letting $x=\frac{1}{e}$ in (2.31) we get (2.32).

Corollary 6. Let $n$ be a integer number such that $n \geq 1$. The following formula

$$
\int_{0}^{1} \frac{d t}{\log ^{n+1}(1 / x t)}=\frac{1}{n !}\left[\frac{(-1)^{n-1} \operatorname{Ei}(\log (x))}{x}+\sum_{m=1}^{n} \frac{(m-1) !(-1)^{n-m}}{\log ^{m}(1 / x)}\right]
$$


is valid for all $0<x<1$. In particular, we get

$$
\gamma=\frac{1}{e}\left[\int_{0}^{1} \frac{d t}{(1-\log (t))^{2}}-1\right]-\sum_{k=1}^{\infty} \frac{(-1)^{k}}{k \cdot k !}
$$

where $\gamma$ is Euler-Mascheroni constant.

Proof. Putting $a=1, b=0$ and $\beta=n$ in (2.35), we thus get

$$
\int_{0}^{1} \frac{d t}{\log ^{n+1}(1 / x t)}=\frac{1}{\Gamma(n+1)} \int_{0}^{\infty} \frac{t^{n} x^{t} d t}{t+1} .
$$

We now make use of the following formula [6, Eq. (11), p. 135]

$$
\int_{0}^{\infty} \frac{t^{n} e^{-p t}}{t+a} d t=(-1)^{n-1} a^{n} e^{a p} \operatorname{Ei}(-a p)+\sum_{m=1}^{n}(m-1) !(-a)^{n-m} p^{-m}, p>0,|\arg (a)|<\pi
$$

with (2.38) we get (2.36). Setting $n=1$ and $x=\frac{1}{e}$ in (2.36), we obtain

$$
\int_{0}^{1} \frac{d t}{(1-\log (t))^{2}}=1+e \operatorname{Ei}(-1) \text {. }
$$

Keeping in mind the above formula with the following equation

$$
\operatorname{Ei}(x)=\gamma+\log |x|+\sum_{k=1}^{\infty} \frac{x^{k}}{k k !}
$$

we get (2.37).

Moreover, in [12, Theorem 2], Karp and Prilepkina found the Mellin transform of the delta neutral $\mathrm{H}$ function when $\mu=-m, m \in \mathbb{N}_{0}$, that is

$$
\frac{\prod_{i=1}^{p} \Gamma\left(A_{i} t+\alpha_{i}\right)}{\prod_{k=1}^{q} \Gamma\left(B_{k} t+\beta_{k}\right)}=\int_{0}^{\rho} H_{q, p}^{p, 0}\left(\left.z\right|_{\left(A_{p}, \alpha_{p}\right)} ^{\left(B_{q}, \beta_{q}\right)}\right) z^{t-1} d t+v \rho^{t} \sum_{k=0}^{m} l_{m-k} t^{k}, \Re(t)>\gamma,
$$

where the coefficient $v$ is defined by

$$
\nu=(2 \pi)^{\frac{p-q}{2}} \prod_{i=1}^{p} A_{i}^{a_{i}-\frac{1}{2}} \prod_{j=1}^{q} B_{j}^{\frac{1}{2}-b_{j}},
$$

and the coefficients $l_{r}$ satisfy the recurrence relation:

$$
l_{r}=\frac{1}{r} \sum_{m=1}^{r} q_{m} l_{r-m}, \text { with } l_{0}=1,
$$

with

$$
q_{m}=\frac{(-1)^{m+1}}{m+1}\left[\sum_{i=1}^{p} \frac{\mathcal{B}_{m+1}\left(a_{i}\right)}{A_{i}^{m}}-\sum_{j=1}^{p} \frac{\mathcal{B}_{m+1}\left(b_{j}\right)}{B_{j}^{m}}\right],
$$

where $\mathcal{B}_{m}$ is the Bernoulli polynomial defined via generating function [26, p. 588]

$$
\frac{t e^{a t}}{e^{t}-1}=\sum_{n=0}^{\infty} \mathcal{B}_{n}(a) \frac{t^{n}}{n !}, \quad|t|<2 \pi \text {. }
$$

Obviously, by repeating the same calculations in Theorem 1 with (2.41) and use the following known formula

$$
\int_{0}^{\infty} t^{\sigma} e^{-\rho t} d t=\frac{\Gamma(\sigma+1)}{\rho^{\sigma+1}} \sigma>-1, \rho>0,
$$

we can deduce the following result:

Theorem 2. Let $\alpha, \beta>-1, \mu=-m, m \in \mathbb{N}_{0}$ and $\sum_{j=1}^{p} A_{j}=\sum_{k=1}^{q} B_{k}$. Then the generalized Volterra function possesses the following integral representation:

$$
V_{p, q}^{\alpha, \beta}\left[\begin{array}{c}
\left(a_{p}, A_{p}\right) \\
\left(\beta_{q}, B_{q}\right)
\end{array} \mid x\right]=\int_{0}^{\rho} H_{q, p}^{p, 0}\left(\left.t\right|_{\left(A_{p}, \alpha_{p}\right)} ^{\left(B_{q}, \beta_{q}\right)}\right) \frac{x^{\alpha} d t}{t \log ^{\beta+1}(1 /(t x))}+\frac{v x^{\alpha}}{\Gamma(\beta)} \sum_{k=0}^{m} \frac{l_{m-k} \Gamma(\beta+k+1)}{[\log (1 /(x \rho))]^{\beta+k+1}},
$$

where $0<x<1$, the coefficients $l_{r}$ are computed by (2.43), $v$ and $\rho$ are defined in (2.42) and (2.9). 
In the next Theorem, we derive a Laplace type integral expression for the function $t^{\lambda-1} V_{p, q}^{\alpha, \beta}[$.$] .$

Theorem 3. Let $\alpha, \beta>-1$ and $\lambda \geq 0$. Then, the function

$$
V_{p+1, q}^{\alpha+\lambda, \beta}\left[\begin{array}{c|c}
\left(a_{p}, A_{p}\right),(\alpha+\lambda, 1) \\
\left(\beta_{q}, B_{q}\right)
\end{array} \mid \frac{1}{x}\right]=x^{-\lambda} V_{p+1, p}^{\alpha, \beta}\left[\begin{array}{c}
\left(a_{p}, A_{p}\right),(\alpha+\lambda, 1) \\
\left(\beta_{q}, B_{q}\right)
\end{array} \mid \frac{1}{x}\right],
$$

possesses the following integral representation

$$
V_{p+1, q}^{\alpha+\lambda, \beta}\left[\begin{array}{c|c}
\left(a_{p}, A_{p}\right),(\alpha+\lambda, 1) \\
\left(\beta_{q}, B_{q}\right)
\end{array} \mid \frac{1}{x}\right]=\int_{0}^{\infty} e^{-x t} t^{\lambda-1} V_{p, q}^{\alpha, \beta}\left[\begin{array}{c}
\left(a_{p}, A_{p}\right) \\
\left(\beta_{q}, B_{q}\right)
\end{array} \mid t\right] d t, x>0
$$

Moreover the function $V_{p+1, q}^{\alpha+\lambda, \beta}\left[\begin{array}{c}\left(a_{p}, A_{p}\right),(\alpha+\lambda, 1) \\ \left(b_{q}, B_{q}\right)\end{array} \mid \frac{1}{x}\right]$ is completely monotonic on $(0, \infty)$.

Proof. Make use the formula (2.44) and straightforward calculation yields

$$
\begin{aligned}
& \left.\int_{0}^{\infty} e^{-x t} t^{\lambda-1} V_{p, q}^{\alpha, \beta}\left[\begin{array}{c}
\left(a_{p}, A_{p}\right) \\
\left(\beta_{q}, B_{q}\right)
\end{array}\right) t\right] d t=\int_{0}^{\infty} e^{-x t} t^{\lambda-1}\left[\int_{0}^{\infty} \frac{\prod_{i=1}^{p} \Gamma\left(A_{i} s+a_{i}\right)}{\prod_{j=1}^{q} \Gamma\left(B_{j} s+b_{j}\right)} \frac{t^{s+\alpha} s^{\beta}}{\Gamma(\beta+1)} d s\right] d t \\
& =\int_{0}^{\infty} \frac{\prod_{i=1}^{p} \Gamma\left(A_{i} s+a_{i}\right)}{\prod_{j=1}^{q} \Gamma\left(B_{j} s+b_{j}\right)} \frac{s^{\beta}}{\Gamma(\beta+1)}\left[\int_{0}^{\infty} e^{-x t} t^{s+\alpha+\lambda-1} d t\right] d s \\
& =\int_{0}^{\infty} \frac{\Gamma(s+\alpha+\lambda) \prod_{i=1}^{p} \Gamma\left(A_{i} s+a_{i}\right)}{\prod_{j=1}^{q} \Gamma\left(B_{j} s+b_{j}\right)} \frac{s^{\beta} x^{-(s+\alpha+\lambda)}}{\Gamma(\beta+1)} d s \\
& =V_{p+1, p}^{\alpha+\lambda, \beta}\left[\begin{array}{c|c}
\left(a_{p}, A_{p}\right),(\alpha+\lambda, 1) & \frac{1}{x} \\
\left(\beta_{q}, B_{q}\right) & \frac{1}{x}
\end{array}\right] .
\end{aligned}
$$

Since the spectral function $t^{\lambda-1} V_{p, q}^{\alpha, \beta}[t]$ being positive, all prerequisites of the Bernstein Characterization Theorem for the completely monotone functions are fulfilled, that is the function $V_{p+1, q}^{\alpha+\lambda, \beta}\left[\begin{array}{c}\left(a_{p}, A_{p}\right),(\alpha+\lambda, 1) \\ \left(\beta_{q}, B_{q}\right)\end{array} \mid \frac{1}{x}\right]$ is completely monotone in the above-mentioned range of the parameters involved.

For some difficulties and wide-spread errors concerning generalization of the Bernstein Characterization Theorem to absolutely monotonic functions cf. [27].

Remark 3. Using the fact that

$$
\left.V_{1,2}^{\alpha, \beta} \underset{(a, A),(\alpha+1,1)}{(a, A)} \mid x\right]=\mu(x, \beta, \alpha)
$$

and using the formula (2.46), we obtain

$$
L\left(x^{\lambda-1} \mu(x, \beta, \alpha)\right)=\int_{0}^{\infty} \frac{\Gamma(t+\alpha+\lambda) t^{\beta} x^{-t-\alpha-\lambda}}{\Gamma(t+\alpha+1) \Gamma(\beta+1)} d t
$$

In particular, for $\lambda=1$, we find [5, formula 8$]$

$$
L(\mu(x, \beta, \alpha))=\int_{0}^{\infty} \frac{t^{\beta} x^{-t-\alpha}}{\Gamma(\beta+1)} d t=\frac{1}{x^{\alpha+1} \log ^{\beta+1}(x)} .
$$

Letting $\alpha=0$ in the above formula, we obtain the equation (7) in [5]. In addition, we set $\beta=0$ and $\lambda=1$ in (2.48) we obtain the formula 6 in [5].

Theorem 4. Let $\alpha, \beta>-1, \eta>0$. Then, we get

$$
\begin{aligned}
L^{-1}\left\{\frac{\log (\xi)}{\xi} V_{p, q-1}^{\alpha, \beta}\left[\begin{array}{c}
\left(a_{p}, A_{p}\right) \\
\left(b_{q-1}, B_{q-1}\right)
\end{array} \mid \frac{1}{\xi}\right]\right\}(x)= & \int_{0}^{\infty} \frac{\prod_{i=1}^{p} \Gamma\left(a_{i}+t A_{i}\right) t^{\beta} \chi^{t+\alpha} \psi(t+\alpha+1)}{\Gamma(\beta+1) \Gamma(\alpha+1+t) \prod_{j=1}^{q-1} \Gamma\left(b_{j}+t B_{j}\right)} d t \\
& -\log (\xi) V_{p, q}^{\alpha, \beta}\left[\underset{\left(b_{q-1}, B_{q-1}\right),(\alpha+1,1)}{\left(a_{p}, A_{p}\right)} \mid x\right] .
\end{aligned}
$$

Proof. We set $\left(b_{q}, B_{q}\right)=(\alpha+\eta+1,1)$ and define the function $F(x)$ by

$$
F(x)=\int_{0}^{\infty} V_{p, q}^{\alpha+\eta, \beta}\left[\begin{array}{c}
\left(a_{p}, A_{p}\right) \\
\left(b_{p}, B_{q}\right)
\end{array} \mid x\right] f(\eta) d \eta
$$


Keeping (2.44) in mind, we get

$$
\begin{aligned}
L(F)(\xi) & =\int_{0}^{\infty} \int_{0}^{\infty} e^{-\xi x} V_{p, q}^{\alpha+\eta, \beta}\left[\begin{array}{l}
\left(a_{p}, A_{p}\right) \\
\left(b_{p}, B_{q}\right)
\end{array} \mid x\right] f(\eta) d \eta d x \\
& =\int_{0}^{\infty} \int_{0}^{\infty} \int_{0}^{\infty} e^{-\xi x} \frac{\prod_{i=1}^{p} \Gamma\left(A_{i} t+a_{i}\right) x^{t+\alpha+\eta} t^{\beta}}{\Gamma(\beta+1) \prod_{j=1}^{q} \Gamma\left(B_{j} t+b_{j}\right)} f(\eta) d \eta d x d t \\
& =\int_{0}^{\infty} \int_{0}^{\infty} \frac{\prod_{i=1}^{p} \Gamma\left(A_{i} t+a_{i}\right) t^{\beta}}{\Gamma(\beta+1) \prod_{j=1}^{q-1} \Gamma\left(B_{j} t+b_{j}\right)}\left(\frac{1}{\xi}\right)^{t+\alpha+\eta+1} f(\eta) d \eta d t \\
& =\xi^{-1} V_{p, q-1}^{\alpha, \beta}\left[\begin{array}{l}
\left(a_{p}, A_{p}\right) \\
\left(b_{q-1}, B_{q-1}\right)
\end{array} \mid 1 / \xi\right] \int_{0}^{\infty}\left(\frac{1}{\xi}\right)^{\eta} f(\eta) d \eta \\
& =V_{p, q-1}^{\alpha, \beta}\left[\begin{array}{l}
\left(a_{p}, A_{p}\right) \\
\left(b_{q-1}, B_{q-1}\right)
\end{array} \mid \frac{1}{\xi}\right] \frac{L f(\log (\xi))}{\xi} .
\end{aligned}
$$

This implies that

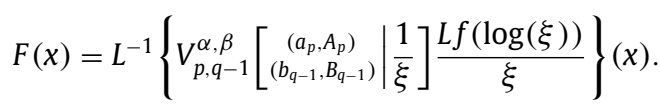

Now, suppose that $f(\eta)=\delta^{\prime}(\eta)$, where $\delta$ is the Dirac delta function. Since $L\left(\delta^{\prime}(\xi)\right)=\xi$, we deduce, by the above formula, that

$$
L^{-1}\left\{V_{p, q-1}^{\alpha, \beta}\left[\begin{array}{c}
\left(a_{p}, A_{p}\right) \\
\left(b_{q-1}, B_{q-1}\right)
\end{array} \mid \frac{1}{x}\right] \frac{L f(\log (\xi))}{\xi}\right\}(\xi)=\int_{0}^{\infty} V_{p, q}^{\alpha+\eta, \beta}\left[\begin{array}{c}
\left(a_{p}, A_{p}\right) \\
\left(b_{p}, B_{q}\right)
\end{array} \mid x\right] \delta^{\prime}(\eta) d \eta .
$$

Combining (2.53)with the following formula

$$
\int_{0}^{\infty} f(t) \delta^{(n)}(t) d t=(-1)^{n} f^{(n)}(0)
$$

we obtain

$$
L^{-1}\left\{V_{p, q-1}^{\alpha, \beta}\left[\begin{array}{c}
\left(a_{p}, A_{p}\right) \\
\left(b_{q-1}, B_{q-1}\right)
\end{array} \mid \frac{1}{x}\right] \frac{L f(\log (x))}{x}\right\}(\xi)=-\lim _{\eta \rightarrow 0} \frac{\partial}{\partial \eta} V_{p, q}^{\alpha+\eta, \beta}\left[\begin{array}{c}
\left(a_{p}, A_{p}\right) \\
\left(b_{p}, B_{q}\right)
\end{array} \mid \xi\right] .
$$

Moreover, we have

$$
\begin{aligned}
\lim _{\eta \rightarrow 0} \frac{\partial}{\partial \eta} V_{p, q}^{\alpha+\eta, \beta}\left[\begin{array}{c}
\left(a_{p}, A_{p}\right) \\
\left(b_{p}, B_{q}\right)
\end{array} \mid \xi\right]= & \log (\xi) V_{p, q}^{\alpha, \beta}\left[\begin{array}{c}
\left(a_{p}, A_{p}\right) \\
\left(b_{q-1}, B_{q-1}\right),(\alpha+1,1)
\end{array} \mid \xi\right] \\
& -\int_{0}^{\infty} \frac{\prod_{i=1}^{p} \Gamma\left(a_{i}+t A_{i}\right) t^{\beta} \xi^{t+\alpha} \psi(t+\alpha+1)}{\Gamma(\beta+1) \Gamma(\alpha+1+t) \prod_{j=1}^{q-1} \Gamma\left(b_{j}+t B_{j}\right)} d t
\end{aligned}
$$

In view of (2.54) and (2.55) we obtain the desired result. The proof of Theorem 4 is complete.

Remark 4. let $q=p+1, a_{i}=b_{i}$ and $A_{i}=B_{i}$ for $i=1, \ldots, p$ in (2.50), we obtain

$$
L^{-1}\left\{\frac{1}{\xi^{\alpha+1} \log ^{\beta}(\xi)}\right\}(x)=\int_{0}^{\infty} \frac{t^{\beta} x^{t+\alpha} \psi(t+\alpha+1)}{\Gamma(\beta+1) \Gamma(\alpha+1+t)} d t-\log (x) \mu(x, \beta, \alpha) .
$$

In particular, for $\beta=0$, we find [5, Eq. (20)]

$$
\frac{x^{\alpha}}{\Gamma(\alpha+1)}=\int_{0}^{\infty} \frac{x^{t+\alpha} \psi(t+\alpha+1)}{\Gamma(t+\alpha+1)} d t-\log (x) v(x, \alpha), \alpha>-1 .
$$

In addition, by using (2.49), we have

$$
L^{-1}\left\{\frac{1}{\xi^{\alpha+1} \log ^{\beta}(\xi)}\right\}(x)=\mu(x, \beta-1, \alpha) .
$$

Keeping in mind (2.58) and (2.56), we obtain (see [4, formula (38)])

$$
\mu(x, \beta-1, \alpha)+\log (x) \mu(x, \beta, \alpha)=\int_{0}^{\infty} \frac{x^{t+\alpha} \psi(t+\alpha+1)}{\Gamma(t+\alpha+1)} d t .
$$

Corollary 7. Suppose that

$$
\alpha \geq 0, \beta>-1,\left(a_{p}, A_{p}\right)=(\alpha, 1) \text { and }\left(b_{q}, B_{q}\right)=(\alpha+\eta+1,1) .
$$

Then the next convolution representation holds

$$
\int_{0}^{\infty} V_{p, q}^{\alpha+\eta, \beta}\left[\begin{array}{c}
\left(a_{p}, A_{p}\right) \\
\left(b_{p}, B_{q}\right)
\end{array} \mid x\right] f(\eta) d \eta=f(\log (x)) *\left(\frac{1}{x} V_{p-1, q-1}^{\alpha, \beta}\left[\begin{array}{c}
\left(a_{p-1}, A_{p-1}\right) \\
\left(b_{p-1}, B_{q-1}\right)
\end{array} \mid x\right]\right) .
$$


Proof. By means of Theorem 3, we have

$$
L\left\{x^{-1} V_{p, q}^{\alpha, \beta}\left[\begin{array}{l|l}
\left(a_{p}, A_{p}\right) & 1 \\
\left(b_{q}, B_{q}\right) & \frac{1}{x}
\end{array}\right]\right\}(s)=V_{p+1, q}^{\alpha, \beta}\left[\begin{array}{l}
\left(a_{p}, A_{p}\right),(\alpha, 1) \\
\left(b_{q}, B_{q}\right)
\end{array} \mid \frac{1}{s}\right] .
$$

So, from the above formula and (2.52) we conclude that (2.60) holds.

\section{Mathieu-type series associated with the generalized Volterra function}

Our aim in this section is to derive some integral representations for a family of convergent Mathieu-type series and its alternating variants with terms containing generalized Volterra functions.

Throughout this section, we adopt the following notation for the real sequence c:

$$
\text { c : } 0<c_{1}<\cdots<c_{n} \uparrow \infty .
$$

It is useful here to consider the function $c: \mathbb{R}_{+} \rightarrow \mathbb{R}_{+}$such that

$$
\left.c(x)\right|_{x \in \mathbb{N}}=\mathbf{c} .
$$

In this section, we investigate the Mathieu-type series $\mathcal{K}$ and its alternating variant $\tilde{\mathcal{K}}$, which are defined by

$$
\mathcal{K}\left(V_{p+1, q}^{\alpha, \beta} ; \mathbf{c} ; r\right)=\sum_{j=1}^{\infty} \frac{c_{j}^{-\lambda}}{\left(c_{j}+r\right)^{\mu}} \cdot V_{p+1, q}^{\alpha, \beta}\left[\begin{array}{c}
\left(a_{p}, A_{p}\right),(\alpha+\lambda, 1) \\
\left(b_{p}, B_{q}\right)
\end{array} \mid \frac{r}{c_{j}}\right],
$$

and

$$
\tilde{\mathcal{K}}\left(V_{p+1, q}^{\alpha, \beta} ; \mathbf{c} ; r\right)=\sum_{j=1}^{\infty} \frac{(-1)^{j-1} c_{j}^{-\lambda}}{\left(c_{j}+r\right)^{\mu}} \cdot V_{p+1, q}^{\alpha, \beta}\left[\begin{array}{c|c}
\left(a_{p}, A_{p}\right),(\alpha+\lambda, 1) \\
\left(b_{p}, B_{q}\right)
\end{array} \mid \frac{r}{c_{j}}\right]
$$

The Laplace integral form of a Dirichlet series was one of most powerful tools in getting closed integral form expressions for the Mathieu-type series $\mathcal{K}$ and its alternating variant $\tilde{\mathcal{K}}$. For c satisfying (3.62), we have

$$
\mathcal{D}_{\mathbf{c}}(x)=\sum_{n=1}^{\infty} e^{-c_{n}^{\eta} x}=x \int_{0}^{\infty} e^{-x t} A_{\eta}(t) d t,
$$

where the so-called counting function $A_{\eta}(t)$ has been found easily in the following manner

$$
A_{\eta}(t)=\sum_{n: a_{n}^{\eta} \geq t} 1=\left[c^{-1}\left(t^{\frac{1}{\eta}}\right)\right]
$$

where $c^{-1}(t)$ is the inverse function of $c(x)$, and $[a]$ is the integer part of a real number $a$. From this, and using the fact that

$$
\left[c^{-1}\left(t^{\frac{1}{\eta}}\right)\right] \equiv 0, t \in\left[0, c_{1}^{\eta}\right)
$$

we find that

$$
\mathcal{D}_{\mathbf{c}}(x)=x \int_{c_{1}^{\eta}}^{\infty} e^{-x t}\left[c^{-1}\left(t^{\frac{1}{\eta}}\right)\right] d t
$$

A comprehensive overview of this technique is in [24,25]. Note important results on inequalities for Mathieu series proved by Zastavnyi in [30,31] and Tomovski et al. in [28,29].

Theorem 5. Let $\alpha, \beta>-1$ and $\lambda, \mu, r>0$. Then for the Mathieu-type power series $\mathcal{K}\left(V_{p+1, q}^{\alpha, \beta} ; \mathbf{c} ; r\right)$ the next integral representation is valid:

$$
\mathcal{K}\left(V_{p+1, q}^{\alpha, \beta} ; \mathbf{c} ; r\right)=K_{\mathbf{c}}^{V}(r, \lambda, \mu+1)+\mu K_{\mathbf{c}}^{V}(r, \lambda+1, \mu),
$$

where

$$
K_{\mathbf{c}}^{V}(r, \lambda, \mu)=\int_{c_{1}}^{\infty} \frac{\left[c^{-1}(x)\right]}{x^{\lambda}(x+r)^{\mu}} V_{p+1, q}^{\alpha, \beta}\left[\begin{array}{c|c}
\left(a_{p}, A_{p}\right),(\alpha+\lambda, 1) \\
\left(b_{p}, B_{q}\right)
\end{array} \mid \frac{r}{x}\right] d x .
$$

Proof. Consider the Laplace transform formula (2.46) of the function $x^{\lambda-1} V_{p, q}^{\alpha, \beta}[$.$] and in view of (2.44) and (3.66), we get$

$$
\begin{aligned}
\mathcal{K}\left(V_{p+1, q}^{\alpha, \beta} ; \mathbf{c} ; r\right) & =\sum_{j=1}^{\infty} \frac{1}{\left(c_{j}+r\right)^{\mu}} \int_{0}^{\infty} e^{-c_{j} s} s^{\lambda-1} V_{p, q}^{\alpha, \beta}\left[\begin{array}{c}
\left(a_{p}, A_{p}\right) \\
\left(b_{p}, B_{q}\right)
\end{array} \mid r s\right] d x \\
& =\frac{1}{\Gamma(\mu)} \sum_{j=1}^{\infty} \int_{0}^{\infty} \int_{0}^{\infty} e^{-c_{j} s-\left(c_{j}+r\right) t} t^{\mu-1} s^{\lambda-1} V_{p, q}^{\alpha, \beta}\left[\begin{array}{c}
\left(a_{p}, A_{p}\right) \\
\left(b_{p}, B_{q}\right)
\end{array} \mid r s\right] d x d t
\end{aligned}
$$




$$
\begin{aligned}
& \left.=\frac{1}{\Gamma(\mu)} \int_{0}^{\infty} \int_{0}^{\infty}\left(\sum_{j=1}^{\infty} e^{-c_{j}(s+t)}\right) e^{-r t} t^{\mu-1} s^{\lambda-1} V_{p, q}^{\alpha, \beta}\left[\begin{array}{c}
\left(a_{p}, A_{p}\right) \\
\left(b_{p}, B_{q}\right)
\end{array}\right) r s\right] d x d t \\
& \left.=\int_{c_{1}}^{\infty} \frac{\left[c^{-1}(x)\right]}{\Gamma(\mu)}\left(\int_{0}^{\infty} e^{-(r+x) t} t^{\mu-1} d t\right)\left(\int_{0}^{\infty} e^{-x s} s^{\lambda} V_{p, q}^{\alpha, \beta}\left[\begin{array}{c}
\left(a_{p}, A_{p}\right) \\
\left(b_{p}, B_{q}\right)
\end{array}\right) r s\right] d s\right) d x \\
& +\int_{c_{1}}^{\infty} \frac{\left[c^{-1}(x)\right]}{\Gamma(\mu)}\left(\int_{0}^{\infty} e^{-(r+x) t} t^{\mu} d t\right)\left(\int_{0}^{\infty} e^{-x s} s^{\lambda-1} V_{p, q}^{\alpha, \beta}\left[\begin{array}{c}
\left(a_{p}, A_{p}\right) \\
\left(b_{p}, B_{q}\right)
\end{array} \mid r s\right] d s\right) d x \\
& =\int_{c_{1}}^{\infty} \frac{\left[c^{-1}(x)\right]}{x^{\lambda+1}(r+x)^{\mu}} V_{p+1, q}^{\alpha, \beta}\left[\begin{array}{l|l}
\left(a_{p}, A_{p}\right),(\alpha+\lambda+1,1) & \mid r \\
\left(b_{q}, B_{q}\right)
\end{array}\right] d s d x \\
& +\mu \int_{c_{1}}^{\infty} \frac{\left[c^{-1}(x)\right]}{x^{\lambda}(r+x)^{\mu+1}} V_{p+1, q}^{\alpha, \beta}\left[\begin{array}{l}
\left(a_{p}, A_{p}\right),(\alpha+\lambda, 1) \\
\left(b_{q}, B_{q}\right)
\end{array} \mid \frac{r}{x}\right] d s d x,
\end{aligned}
$$

which proves the Theorem 5 .

Obviously, by repeating the same calculations as above and using the formula (see [25, Eq. (13)])

$$
\tilde{D}_{\mathbf{c}}(y)=\sum_{j=1}^{\infty}(-1)^{j-1} e^{-c_{j} y}=y \int_{c_{1}}^{\infty} e^{-y x} \sin ^{2}\left(\frac{\pi}{2}\left[c^{-1}(x)\right]\right) d x,
$$

we can deduce the following result for the alternating Mathieu-type power series $\tilde{\mathcal{K}}$.

Theorem 6. Let $\alpha, \beta>-1$ and $\lambda, \mu, r>0$. Then for the alternating Mathieu-type power series $\tilde{\mathcal{K}}\left(V_{p+1, q}^{\alpha, \beta} ; \mathbf{c} ; r\right)$ the next integral representation is valid:

$$
\tilde{\mathcal{K}}\left(V_{p+1, q}^{\alpha, \beta} ; \mathbf{c} ; r\right)=\tilde{K}_{\mathbf{c}}^{V}(r, \lambda, \mu+1)+\mu \tilde{K}_{\mathbf{c}}^{V}(r, \lambda+1, \mu),
$$

where

$$
\tilde{K}_{\mathbf{c}}^{V}(r, \lambda, \mu)=\int_{c_{1}}^{\infty} \frac{\sin ^{2}\left(\frac{\pi}{2}\left[c^{-1}(x)\right]\right)}{x^{\lambda}(x+r)^{\mu}} V_{p+1, q}^{\alpha, \beta}\left[\begin{array}{c}
\left(a_{p}, A_{p}\right),(\alpha+\lambda, 1) \\
\left(b_{p}, B_{q}\right)
\end{array} \mid \frac{r}{x}\right] d x .
$$

\section{Conclusion}

Our present investigation was motivated essentially by the fact that the Volterra function play an important role in a variety of fields of mathematics, namely in the theory of definite integrals, integral equations and prime numbers. In recent years, such studies have attracted a large number of researchers who made use in a large variety of problems associated with the properties of other special functions, for example, Apelblat has proved that the infinite integrals with respect to the order of the Bessel and related functions can be developed into series of the Volterra functions and some infinite integrals of gamma and polygamma functions are expressible in terms of the Volterra functions. In this paper, we first introduced extension of the Volterra function in term of a ratio of gamma functions, we then proved its various properties such as integral representations when their terms contain the Fox $\mathrm{H}$-function. In particular, we have presented several other properties: infinite integral of some special functions are expressed in terms of the generalized Volterra functions, closed-form integral representations for a family of convergent Mathieu-type series defined in terms of generalized Volterra functions are established, and some generalization of some results proved in the literature for the classical Volterra function are given.

\section{Acknowledgment}

The authors like to thank Prof. Alexander Apelblat for providing us with the copy of his book on Volterra functions, it was very useful for the preparation of this paper

\section{References}

[1] A. Erdelyi, W. Magnus, F. Oberhettinger, F.G. Tricomi, Higher Transcendental Functions, McGraw-Hill, New York, 1955 , pp. $217-227$.

[2] M.M. Dzhrbashyan, Integral Transforms and Functions Representations in the Complex Domain, Nauka, Moscow, 1966. (in Russian).

[3] A. Apelblat, Volterra Functions, Nova Science Publ. Inc., New York, 2008.

[4] A. Apelblat, Integral Transforms and Volterra Functions, Nova Science Publ. Inc., New York, 2010.

[5] A. Apelblat, Some integrals of gamma, polygamma and volterra functions, IMA J. Appl. Math. 34 (1985) $173-186$.

[6] A. Erdelyi, W. Magnus, F. Oberhettinger, F.G. Tricomi, Tables of Integral Transforms, Vol. 1, McGraw-Hill, New York, 1954.

[7] R. Garrappa, F. Mainardi, On volterra functions and ramanujan integrals, 2016.ArXiv:1610.01491v1. 21

[8] A. Baricz, Turan type inequalities for hypergeometric functions, Proc. Am. Math. Soc. 136 (9) (2008) $3223-3229$.

[9] A. Baricz, Functional inequalities involving bessel and modified bessel functions of the first kind, Expos. Math. 26 (2008) $279-293$.

[10] D.E. Karp, E. Prilepkina, Hypergeometric differential equation and new identities for the coefficients of nørlund and bühring, Symm. Integr. Geom.: Methods Appl. SIGMA 12 (052) (2016) 23.

[11] D.E. Karp, E. Prilepkina, Completely monotonic gamma ratio and infinitely divisible H-function of fox, Comput. Methods Funct. Theory 16 (2016) 135-153. 
[12] D.E. Karp, E. Prilepkina, Some new facts concerning the delta neutral case of Foxs H function, Comput. Methods Funct. Theory 17 (2) (2017) $343-367$.

[13] D.E. Karp, S.M. Sitnik, Log-convexity and log-concavity of hypergeometric-like functions, J. Math. Anal. Appl. 364 (2) (2010) 384-394.

[14] D.E. Karp, S.M. Sitnik, Inequalities and monotonicity of ratios for generalized hypergeometric function, J. Approx. Theory 161 (2009) 337-352.

[15] A.M. Mathai, A Handbook of Generalized Special Functions for Statistical and Physical Sciences, Oxford University Press, Oxford, 1993.

[16] A.M. Mathai, R.K. Saxena, H.J. Haubold, The H-functions: Theory and applications, Springer, 2010.

[17] K. Mehrez, S.M. Sitnik, Functional inequalities for the Mittag-Lefller functions, Results Math. 72 (1) (2017) $703-714$

[18] K. Mehrez, S.M. Sitnik, On monotonicity of ratios of some hypergeometric functions, Sib. Electron. Math. Rep. 13 (2016) 260-268.

[19] K. Mehrez, S.M. Sitnik, On monotonicity of ratios of some q-hypergeometric functions, Mat. Vesn. 68 (3) (2016) $225-231$.

[20] K. Mehrez, S.M. Sitnik, Proofs of some conjectures on monotonicity of ratios of Kummer, gauss and generalized hypergeometric functions, Analysis (De Gruyter) 36 (4) (2016) 263-268.

[21] K. Mehrez, Functional inequalities for the wright functions, Integral Trans. Special Funct. 28 (2) (2017) 130-144.

[22] K. Mehrez, New integral representation for the fox-wright functions and their applications, 2017b. ArXiv:1711.08368. 15

[23] D.S. Mitrinović, Analytic Inequalities, Springer-Verlag, Berlin, 1970.

[24] T.K. Pogány, Integral representations of a series which includes the mathieu a-series, J. Math. Anal. Appl. 296 (1) (2004) $309-313$.

[25] T.K. Pogány, H.M. Srivastava, Z. Tomovski, Some families of Mathieu a-series and alternative Mathieu a-series, Appl. Math. Comput. 179 (1) (2006) 69-108.

[26] F.W.J. Olver, D.W. Lozier, R.F. Boisvert, C.W. Clark (Eds.), NIST Handbook of Mathematical Functions, Cambridge University Press, Cambridge, 2010.

[27] S.M. Sitnik, A note on the main theorem for absolutely monotonic functions, 2012, ArXiv:1202.1210v2. (updated 2014). 4

[28] Z. Tomovski, K. Mehrez, Some families of generalized Mathieu-type power series, associated probability distributions and related inequalities involving complete monotonicity and log-convexity, Math. Ineq. Appl. 20 (4) (2017) 973-986.

[29] H.M. Srivastava, K.K. Mehrez, Z. Tomovski, New inequalities for some generalized Mathieu type series and the Riemann zeta function, J. Math. Ineq. 12 (1) (2018) 163-174.

[30] V.P. Zastavnyi, Inequalities for the Mathiue series and positive defitness, Anal. Math. 37 (4) (2011) 289-318.

[31] V.P. Zastavnyi, Positive definite functions and some problems in analysis, Thesis, Donetsk, $2011 \mathrm{~b}, \mathrm{p} .335$. 